\title{
Caracterização de Tubo e Adesivo Utilizados em Tubulações de Plástico Reforçado com Fibras de Vidro Aplicados em Plataformas Marítimas
}

\author{
Maikon C. R. Pessanha, Dylmar P. Dias \\ Laboratório de Engenharia Civil, UENF \\ Marcello Filgueira, Ronaldo P. R. Paranhos \\ Laboratório de Materiais Avançados, UENF
}

\section{Otávio L. de Farias \\ Petrobras}

Resumo: Na indústria de exploração e produção de petróleo, principalmente no setor offshore, os plásticos reforçados por fibras de vidro (PRFV) têm apresentado grande destaque. Isso se deve à intrínseca capacidade destes de resistir à corrosão em ambientes salinos, bem como serem materiais que apresentam elevada resistência mecânica específica. $\mathrm{O}$ presente trabalho buscou caracterizar tubo e adesivo utilizados em plataformas marítimas. Para tanto, as técnicas utilizadas foram: espectroscopia na região do infravermelho com transformada de Fourier (FTIR), análise termogravimétrica (TGA), análise termodinâmico-mecânica (DMTA) e temperatura de amolecimento de Vicat. Amostras do tubo de PRFV e dos componentes do adesivo (denominados de A e B) foram estudadas individualmente. Além disso, foram confeccionadas misturas, em massa, nas seguintes proporções: 40\%A-60\%B, 50\%A-50\%B e 60\%A-40\%B. Foi possível determinar a composição e a estrutura química dos materiais, além do comportamento mecânico à degradação térmica. Foi verificado que adesivo e tubo apresentaram desempenho satisfatório à temperatura ambiente quanto à degradação e à rigidez. Por outro lado, quando submetido ao calor, principalmente os adesivos, apresentaram queda brusca de desempenho.

Palavras-chave: Adesivos, indústria offshore, plataforma marítima, PRFV, temperatura.

\section{Characterization of Pipes and Adhesives of Glassfiber Reinforced Plastics Used in Offshore Platforms}

Abstract: Glassfiber reinforced plastics (GFRP) have been applied in the offshore industry owing to their high resistance to corrosion and high specific mechanical strength. The present work aimed at characterizing pipes and adhesives used in offshore platforms in order to evaluate the thermo-mechanical performance of these materials. The techniques used were: Fourier transform infrared spectroscopy (FTIR), thermogravimetry analysis (TGA), dynamic mechanical thermal analysis (DMTA) and Vicat softening temperature. Specimens of GFRP pipe and adhesive components (referred to as A and B) were evaluated individually. The adhesive samples were made in the following ratios (\% weight): $40 \% \mathrm{~A}-60 \% \mathrm{~B}, 50 \% \mathrm{~A}-50 \% \mathrm{~B}$ and $60 \% \mathrm{~A}-40 \% \mathrm{~B}$. The results showed it to be possible to determinate the composition and structure of the materials, as well as the mechanical behavior regarding thermal degradation. The adhesive and pipe exhibited satisfactory performance at room temperature, with regard to degradation and stiffness. Nevertheless, at higher temperatures, both materials, especially the adhesives, presented abrupt strength reduction.

Keywords: Adhesives, GFRP, offshore industry, temperature effect.

\section{Introdução}

A utilização de tubulações de plásticos reforçados por fibras de vidro (PRFV) tem apresentado grande destaque na indústria de exploração e produção de petróleo ${ }^{[1]}$, principalmente no setor offshore, pois tal material apresenta alta resistência à corrosão e à abrasão ${ }^{[2]}$, além do baixo peso quando comparado às tubulações metálicas com propriedades similares, reduzindo assim o peso das plataformas marítimas, além de elevada resistência mecânica ${ }^{[2-4]}$. Estas características implicam menor incidência de manutenção, pintura e substituição, auxiliando na diminuição dos tempos de parada das unidades produtivas ${ }^{[2]}$. Além de serem utilizadas em plataformas de perfuração e produção de petróleo e em sistemas de tratamento de efluentes, no Brasil, estas também são utilizadas em sistemas de captação de água para refrigeração, injeção e combate a incêndio ${ }^{[5,6]}$.

Autor para correspondência: Maikon Caetano Ramos Pessanha, Laboratório de Engenharia Civil, UENF, Av. Alberto Lamego 2000, Pq. Califórnia, CEP: 28013-602, Campos dos Goytacazes, RJ, Brasil.E-mail: maikon@uenf.br 
As juntas nestes tipos de tubulação podem ser coladas, laminadas ou mecânicas ${ }^{[5]}$. Sendo assim, o presente trabalho visou caracterizar tubo e adesivo utilizados em plataformas marítimas. Para tanto, foram utilizadas técnicas de espectroscopia na região do infravermelho com transformada de Fourier (FTIR), análise termogravimétrica (TGA), análise termodinâmico-mecânica (DMTA) e temperatura de amolecimento de Vicat.

\section{Materiais e Métodos}

Nas análises, foram utilizados materiais provenientes de um fornecedor internacional, onde foram extraídas amostras dos tubos de PRFV e do adesivo bi-componente, conforme informações do fabricante. As amostras de tubo e os componentes do adesivo (denominados de $\mathrm{A}$ e $\mathrm{B}$, respectivamente, resina e catalisador) foram avaliados individualmente, assim como misturas, em massa, nas seguintes proporções: $40 \% \mathrm{~A}-60 \% \mathrm{~B}, 50 \% \mathrm{~A}-50 \% \mathrm{~B}$ e $60 \% \mathrm{~A}-40 \% \mathrm{~B}$. A Tabela 1 apresenta a nomenclatura adotada para as amostras analisadas.

Nas análises por espectroscopia no infravermelho com transformada de Fourier (FTIR), utilizou-se um espectrofotômetro da Thermo Nicolet tipo Nexus 4700 . Os espectros foram obtidos com leitura entre 4000 e $400 \mathrm{~cm}^{-1}, 32$ repetições e resolução de $4 \mathrm{~cm}^{-1}$. Os espectros das amostras CPAA e CPAB foram obtidos depositando-se uma pequena quantidade destas amostras sobre pastilhas de $\mathrm{KBr}$. Para as demais amostras os espectros foram obtidos segundo duas condições distintas: prensagem em pastilhas de material raspado com cristais de $\mathrm{KBr}$ e pirólise das amostras e com análise do produto destas.

Para as análises termogravimétricas (TGA) foi utilizado um módulo termogravimétrico Hi-Res TGA 2950 (TA Instruments) acoplado a um analisador térmico TA2000 (TA Instruments), com taxa de aquecimento de $10{ }^{\circ} \mathrm{C} / \mathrm{min}$ e atmosfera dinâmica de $\mathrm{N}_{2}$ com vazão de $50 \mathrm{~mL} / \mathrm{min}$. Para as análises, foi utilizado suporte de amostra de alumina na faixa de temperatura entre 0 e $900{ }^{\circ} \mathrm{C}$.

As análises termodinâmico-mecânicas (DMTA) foram efetuadas em um reogoniômetro Ares da Rheometrics, com modo de torção, deformação de $0,1 \%$, freqüência de oscilação de $1 \mathrm{~Hz}$ e taxa de aquecimento de $3{ }^{\circ} \mathrm{C} / \mathrm{min}$.

A temperatura de amolecimento de Vicat foi determinada segundo as especificações da ASTM D1525 $5^{[7]}$. Nesta análise, uma agulha de ponta chata (com área de 1,0 $\pm 0,015 \mathrm{~mm}^{2}$ ) é disposta em contato direto com a amostra. As forças aplica-

Tabela 1. Nomenclatura das amostras analisadas.

\begin{tabular}{lc}
\hline \multicolumn{1}{c}{ Amostras } & Nomenclatura \\
\hline Componente A (resina) & CPAA \\
Componente B (catalisador) & CPAB \\
$40 \%$ A-60\%B & CPA40A60B \\
$50 \%$ A-50\%B & CPA50A50B \\
$60 \%$ A-40\%B & CPA60A40B \\
Tubo de PRFV & CPAT \\
\hline
\end{tabular}

das foram de $10 \pm 0,2 \mathrm{~N}$ e $50 \pm 1,0 \mathrm{~N}$ e a taxa de aquecimento foi fixada em $120 \pm 10^{\circ} \mathrm{C} / \mathrm{h}$. Como meio de transferência de calor foi utilizado um banho de óleo de silicone em um equipamento HDT 6 VICAT P/N 6921 da CEAST.

Para todas as análises, foram preparados corpos-de-prova com espessura variando entre 3 e $6,5 \mathrm{~mm}$, tendo largura de cerca de $12 \mathrm{~mm}$ e comprimento de $60 \mathrm{~mm}$. Todas as amostras foram lixadas para minorar os defeitos de superfície. Cabe salientar que para todas as análises foram utilizados de 3 a 5 corpo de prova.

\section{Resultados e Discussão}

\section{Espectroscopia no infravermelho com transformada de Fourier (FTIR)}

A análise dos espectros obtidos na análise de FTIR permitiu a determinação preliminar da estrutura e dos grupamentos químicos presentes nas amostras. As Figuras 1, 2 e 3 apresentam os espectros das amostras CPAA, CPAB e CPAT, respectivamente. Salienta-se que, nas Figuras 1 e 2, a curva superior é o padrão e a inferior refere-se à amostra analisada. Por outro lado, na Figura 3 as duas curvas superiores são padrões e as inferiores referem-se à amostra.

Da amostra CPAA, a intensidade das absorções apresenta-se elevada, prejudicando de certa forma a identificação

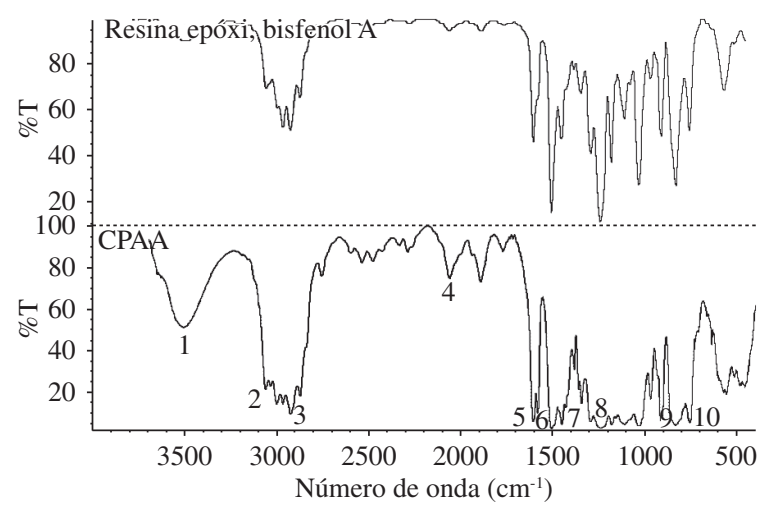

Figura 1. Espectros no infravermelho obtidos da amostra CPAA e um similar de resina bisfenol A.

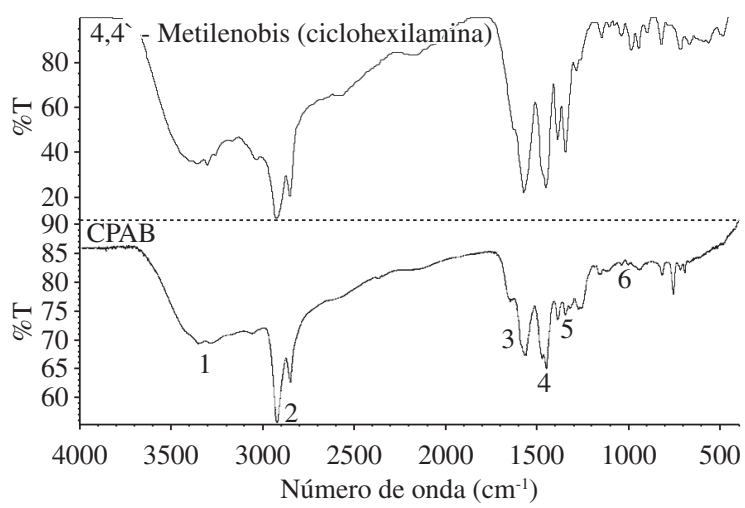

Figura 2. Espectros no infravermelho obtidos da amostra CPAB e um similar 4,4' Metileno bis(ciclohexilamina). 


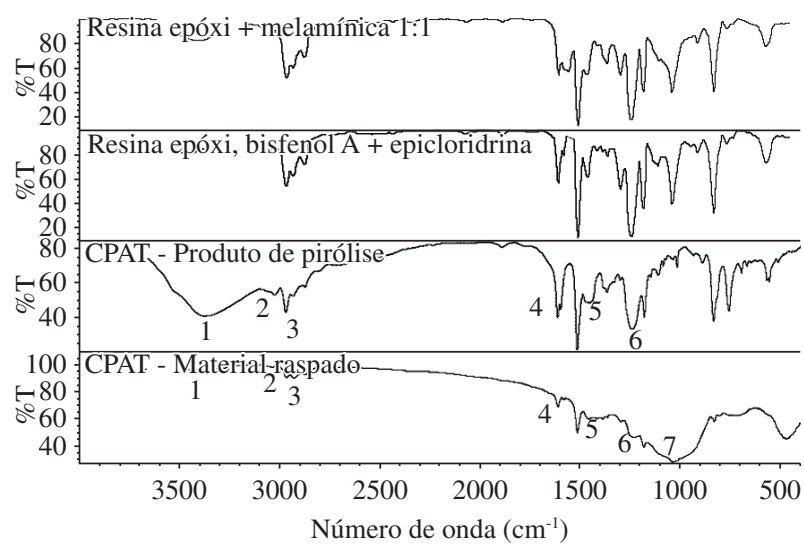

Figura 3. Espectros no infravermelho obtidos da amostra CPAT (material raspado e produto de pirólise) e substâncias similares no banco de dados.

da estrutura química. Todavia, as bandas representam possíveis deformações axiais de $\mathrm{O}-\mathrm{H}$ (banda 1: $3508 \mathrm{~cm}^{-1}$ ), de C-H de aromáticos (banda 2: $3058 \mathrm{~cm}^{-1}$ ) e alifáticos $\mathrm{CH}_{2} \mathrm{e}$ $\mathrm{CH}_{3}$ simétricos e assimétricos (banda 3: 2966-2838 $\mathrm{cm}^{-1}$ ), de $\mathrm{C}=\mathrm{C}$ (banda 5: $1608 \mathrm{~cm}^{-1}$ ), e de C-O (banda 8: $1240 \mathrm{~cm}^{-1}$ ), além de harmônicas ou bandas de combinação de aromáticos (banda 4: 2289-1772 $\mathrm{cm}^{-1}$ ), deformação angular no plano de $\mathrm{CH}_{2}$ sobreposta à deformação axial no plano de $\mathrm{C}=\mathrm{C}$ (banda 6: $1453 \mathrm{~cm}^{-1}$ ), deformação angular no plano de $\mathrm{CH}_{3}$ (banda 7: $1345 \mathrm{~cm}^{-1}$ ) e deformação angular fora do plano de C-H (bandas 9: 831 e 10: $756 \mathrm{~cm}^{-1}$ ). Dessa forma, pode-se estimar que a amostra CPAA é composta por ligações $\mathrm{C}-\mathrm{H}$ alifáticas e aromáticas e por grupos hidroxila $(\mathrm{O}-\mathrm{H})$. Buscas em banco de dados com espectros de distintas substâncias apresentaram sobreposição das bandas de absorção superior a 79\% com as bandas de uma resina epóxi, possivelmente, bisfenol A. Espectros de FTIR obtidos por Pires et al. ${ }^{[12]} \mathrm{em}$ amostras de resina epóxi tipo bisfenol A (DGEBA) curada com dietilenotriamina (DETA) indicaram as presenças das bandas 1 $\left(3500-3200 \mathrm{~cm}^{-1}\right), 2\left(3100-3000 \mathrm{~cm}^{-1}\right), 3\left(3000-2840 \mathrm{~cm}^{-1}\right)$ e $8\left(1250 \mathrm{~cm}^{-1}\right)$, reforçando a possibilidade da amostra apresentada no presente trabalho de ser um bisfenol A.

Ainda segundo Pires et al. ${ }^{[12]}$ a resina epóxi é caracterizada pelo grupamento epóxi em três bandas características, nas regiões de cerca de $1250 \mathrm{~cm}^{-1}$ corresponde à deformação axial simétrica do anel epóxi, em que todas as ligações do anel expandem-se e contraem-se em fase. Outra banda característica esta na faixa $916 \mathrm{~cm}^{-1}$ que pode ser atribuída à deformação assimétrica do anel, na qual a ligação C-C se expande ocorrendo contrações das ligações C-O. A terceira banda característica está em $830 \mathrm{~cm}^{-1}$ associada à deformação simétrica no plano da ligação C-O-C.

Semelhantemente à amostra CPAA, a amostra CPAB (Figura 2) apresenta bandas referentes à deformação axial de O-H (banda 1: 3349-3283 $\mathrm{cm}^{-1}$ ), contudo a amostra CPAB apresenta sobreposição desta com deformação axial de N-H. Outras similaridades são deformações axiais de alifáticos $\mathrm{CH}_{2}$ (banda 2: 2920-2848 $\mathrm{cm}^{-1}$ ) e de C-O (banda 5: $1274 \mathrm{~cm}^{-1}$ ), além de deformação angular no pla- no de $\mathrm{CH}_{2}$ (banda 4: $1449 \mathrm{~cm}^{-1}$ ). O diferencial da amostra apresentada na Figura 2 é a presença de deformação angular simétrica no plano de N-H (banda 3: $1647 \mathrm{~cm}^{-1}$ ) e deformação axial de C-N (banda 6: $1037 \mathrm{~cm}^{-1}$ ). Destes resultados preliminares, a amostra CPAB é composta basicamente de ligações $\mathrm{C}-\mathrm{H}$ alifáticas, por grupamentos amina $(\mathrm{N}-\mathrm{H})$ e provável presença de hidroxila $(\mathrm{O}-\mathrm{H})$, uma vez que a banda característica da mobilidade deste grupamento está sobreposto à deformação axial de N-H. Utilizando-se banco de dados de compostos conhecidos ficou evidente uma sobreposição superior a $85 \%$ entre as bandas de absorção da amostra analisada e de 4,4' Metileno bis(ciclohexilamina). Lançando mão dos espectros de FTIR obtidos por Pires et al. ${ }^{[12]} \mathrm{em}$ amostras de resina epóxi tipo bisfenol A (DGEBA) curada com dietilenotriamina (DETA) foi verificada somente a presença das bandas $2\left(1650-1580 \mathrm{~cm}^{-1}\right)$ e $3\left(3000-2840 \mathrm{~cm}^{-1}\right)$ visto o catalisador obtido no presente trabalho difere daquele.

Os espectros das amostras CPA40A60B, CPA50A50B e CPA60A40B apresentaram sobreposição das bandas de absorção das amostras CPAA e CPAB, com diferenças apenas nas intensidades das bandas.

Da mesma forma que a amostra CPAA (Figura 1), a amostra CPAT (Figura 3) apresenta algumas similaridades, como possíveis deformações axiais de $\mathrm{O}-\mathrm{H}$ (banda $1: 3372 \mathrm{~cm}^{-1}$ ), de aromáticos C-H (banda 2: $3022 \mathrm{~cm}^{-1}$ ), de alifáticos $\mathrm{CH}_{2}$ e $\mathrm{CH}_{3}$ simétricos e assimétricos (banda 3: 2963-2838 $\mathrm{cm}^{-1}$ ), de $\mathrm{C}=\mathrm{C}$ (banda 4: $1610 \mathrm{~cm}^{-1}$ ) e de C-O (banda 6: $1297 \mathrm{~cm}^{-1}$ ), além de deformação angular no plano de $\mathrm{CH}_{2}$ (banda 5: $1457 \mathrm{~cm}^{-1}$ ). A similaridade da amostra CPAT (Figura 3) com a amostra CPAB (Figura 2) está em relação aos compostos referentes às bandas $1,3,5$ e 6 . O grande diferencial da amostra de tubo para com os componentes do adesivo é a presença de deformação axial de $\mathrm{Si}-\mathrm{O}$ (banda 7: $1035 \mathrm{~cm}^{-1}$ ), provavelmente devido à presença de silicato na forma de fibras de vidro e/ou material de enchimento, conforme observado por SánchezSoto et al. ${ }^{[8]}$. No produto de pirólise, a banda referente à carga inorgânica não foi observada. Mais uma vez, efetuando-se buscas em bancos de dados contendo espectros de diversas substâncias, foi verificada sobreposição regular em torno de 69\% das bandas de absorção da amostra CPAT com resina epóxi tipo bisfenol A, curada com epicloridrina, ou de resina epóxi curada com melamínica na relação 1:1. As semelhanças entres os espectros obtidos por Pires et al. ${ }^{[12]} \mathrm{e}$ da amostra CPAT foram referentes as bandas $1\left(3500-3200 \mathrm{~cm}^{-1}\right), 2$ $\left(3100-3000 \mathrm{~cm}^{-1}\right), 3\left(3000-2840 \mathrm{~cm}^{-1}\right)$ e $5\left(1500-1400 \mathrm{~cm}^{-1}\right)$ indicando a grande possibilidade da resina do material ser um bisfenol A com indefinição apenas do catalisador.

\section{Análise termogravimétrica (TGA)}

As Figuras 4 a 6 apresentam os resultados da análise termogravimétrica para as amostras CPAA, CPAB, CPAT, respectivamente, além das misturas dos componentes do adesivo nas diferentes proporções.

O comportamento da amostra CPAA pode ser verificado na Figura $4 \mathrm{a}$, onde se percebe que até $215^{\circ} \mathrm{C}$ o componente 


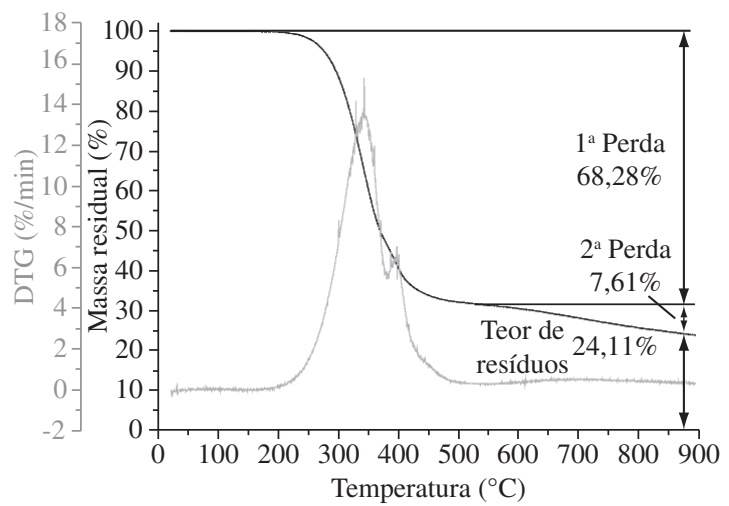

(a)

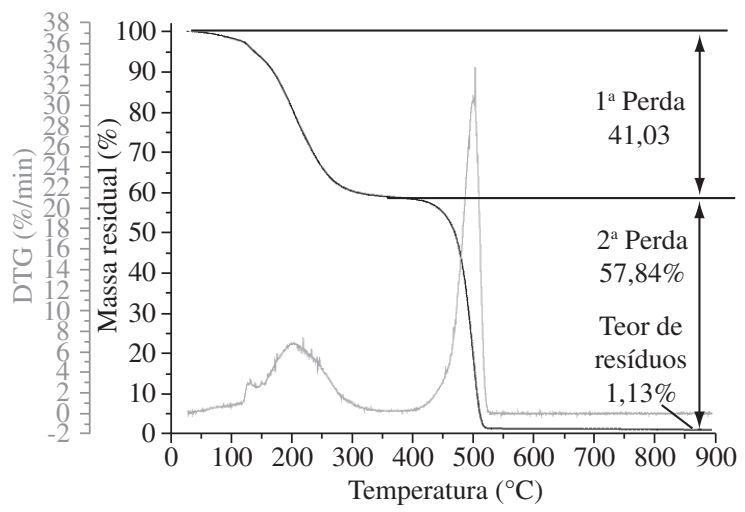

(b)

Figura 4. Curvas TGA e DTG das amostras: a) CPAA; e b) CPAB.

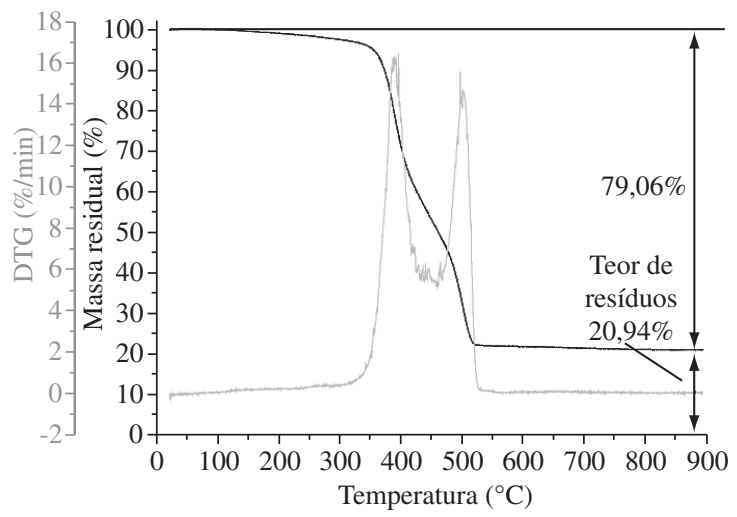

(a)

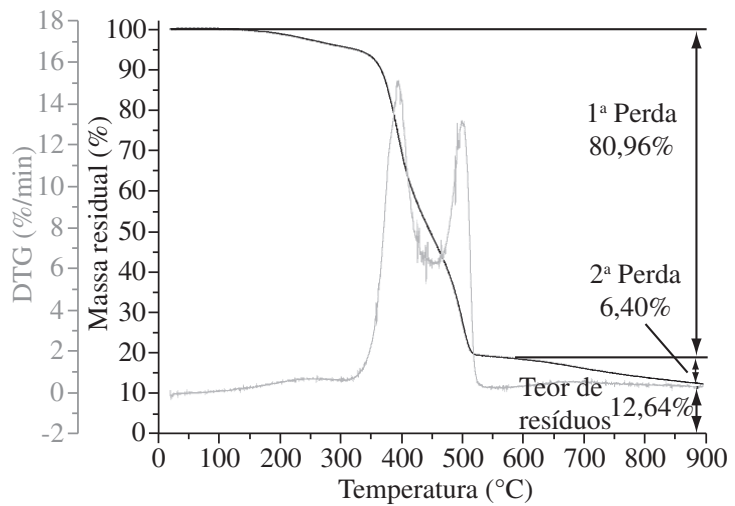

(b)

Figura 5. Curvas TGA e DTG das amostras: a) CPA40A60B; e b) CPA50A50B.

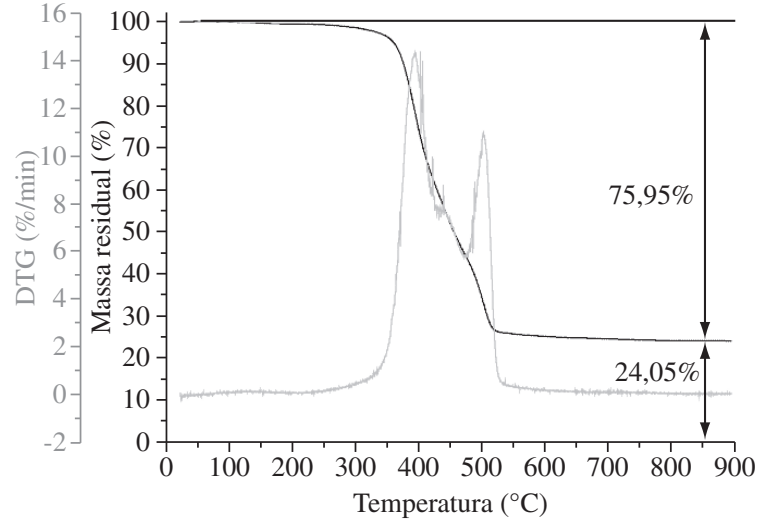

(a)

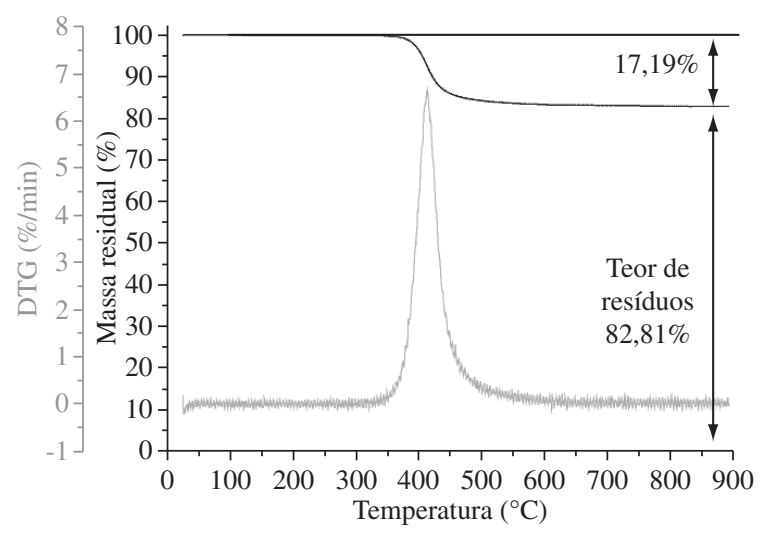

(b)

Figura 6. Curvas TGA e DTG das amostras: a) CPA60A40B; e b) CPAT.

A do adesivo não apresentou nenhuma variação significativa de massa. Entre 215 e $510{ }^{\circ} \mathrm{C}$, a amostra apresentou uma $1^{\text {a }}$ perda $(68,28 \%)$ subdivida em dois eventos térmicos, como pode ser verificado na curva DTG. As temperaturas de decomposição, associadas aos eventos térmicos supracitados, foram 341 e $397^{\circ} \mathrm{C}$. Entre 510 e $550^{\circ} \mathrm{C}$, nota-se um pequeno patamar com uma perda lenta de massa $(7,61 \%)$ a partir de $550{ }^{\circ} \mathrm{C}$ até o fim da análise. Ao final da análise, foi verificado um teor de resíduos de $24,11 \%$, provavelmente devido à quantidade de carga possivelmente inorgânica, que por sua vez não ficou evidenciada nos resultados de FTIR (Figura 1).

Com relação ao comportamento do catalisador, a Figura 4b evidencia que a amostra CPAB perde massa praticamente desde o início da análise, onde até $335^{\circ} \mathrm{C}$ ocorre uma $1^{\text {a }}$ perda (41,03\%) com um único evento térmico com uma temperatura de decomposição de $210{ }^{\circ} \mathrm{C}$. Entre 335 e $400{ }^{\circ} \mathrm{C}$, ocorre um pequeno patamar, todavia a partir de 400 até $530{ }^{\circ} \mathrm{C}$ ocorre uma perda significativa de massa da amostra $(57,84 \%)$. Ao final, obteve-se uma massa residual de $1,13 \%$. Assim, pode-se dizer que o componente $\mathrm{B}$ do adesivo possui baixa quantidade de carga não identificável nos resultados de FTIR (Figura 2). Ao estudarem a diferença de estabilidade térmica entre um bisfenol A, sem e com catalisador à base de amina, Delor-Jestin et al. ${ }^{[9]}$ verificaram que o último permite uma certa estabilidade 
a $400{ }^{\circ} \mathrm{C}$, enquanto o primeiro perdia quase $90 \%$ de sua massa inicial a $200{ }^{\circ} \mathrm{C}$. Os autores verificaram também que o teor de resíduo para todas as amostras variou entre 1 e $3 \%$, ou seja, bem próximo ao encontrado no presente trabalho.

Com relação às misturas adesivas (CPA40A60B, CPA50A50B e CPA60A40B), as Figuras 5a,b e 6a apresentam as curvas termogravimétricas obtidas. Percebe-se que as diferenças estequiométricas não foram suficientes para influenciar qualitativamente nos resultados, pois as amostras não apresentaram perda significativa de massa entre 145 e $165^{\circ} \mathrm{C}$. Entre 150 e $527^{\circ} \mathrm{C}$, as amostras apresentaram uma perda média de $78,66 \%$ de massa em dois eventos térmicos (temperatura de decomposição por volta de 390 e $500{ }^{\circ} \mathrm{C}$ ), podendo-se dizer que o primeiro está associado ao componente $\mathrm{A}$ e o segundo ao B, todavia, deve-se destacar que houve uma reação química no momento que os dois componentes foram misturados e outras com o seu aquecimento. A partir de $530^{\circ} \mathrm{C}$, as amostras apresentaram um patamar até o final da análise, exceto a amostra CPA50A50B, que apresentou uma perda adicional e lenta de 6,40\% atribuída provavelmente a alguma matriz polimérica remanescente. Ao final das análises as amostras apresentaram uma massa residual média de 19,21\%.

A Figura $6 \mathrm{~b}$ apresenta o comportamento da amostra CPAT, onde se nota que até $362{ }^{\circ} \mathrm{C}$ não há perda de massa. Entre 362 e $504^{\circ} \mathrm{C}$, ocorre uma única perda de massa $(17,19 \%)$ em um único evento térmico $\left(413^{\circ} \mathrm{C}\right)$ correspondente a degradação da matriz polimérica. Até o final da análise $\left(900^{\circ} \mathrm{C}\right)$, a amostra apresentou um alto teor de resíduo $(82,81 \%)$ devido à presença de carga inorgânica na forma de fibras de vidro e/ou material de enchimento, conforme também visualizado nos espectros de FTIR. Avaliando curvas de TGA de fibras de vidro, epóxi e epóxi reforçado com 2,5 e $5 \%$ de fibras de vidro, Alonso et al. ${ }^{[10]}$ notaram que à medida que se incorporam fibras na matriz polimérica há uma diminuição de perda de massa inicial, visto que estas fibras apresentam menos de 5\% de perda de massa até $900{ }^{\circ} \mathrm{C}$, em conformidade com os resultados aqui apresentados referentes ao alto teor de resíduo remanescente no compósito.

Verifica-se neste ponto que todas as amostras de adesivo (CPA40A60B, CPA50A50B e CPA60A40B) iniciam degradação térmica a temperaturas muito inferiores que o tubo (CPAT), isto indica que em uma junta colada tubo/adesivo/ tubo sob temperatura da ordem de $145{ }^{\circ} \mathrm{C}$ poderá ocorrer algum tipo de falha comprometendo a operacionalidade da mesma.

\section{Análise termodinâmico-mecânica (DMTA)}

As Figuras 7a,b apresentam, respectivamente, as curvas G' e $\tan \delta$, sendo ambas em função da temperatura.

A Figura 7a mostra os valores de módulo de cisalhamento das amostras em função da temperatura. Nota-se que na temperatura ambiente a amostra CPAT apresentou módulo de cisalhamento igual a 2,5 GPa, e que a amostra adesiva que mais se aproximou foi CPA60A40B (1,0 GPa). Salienta-se que o fabricante prescreve a mistura de um kit em volume. Todavia,

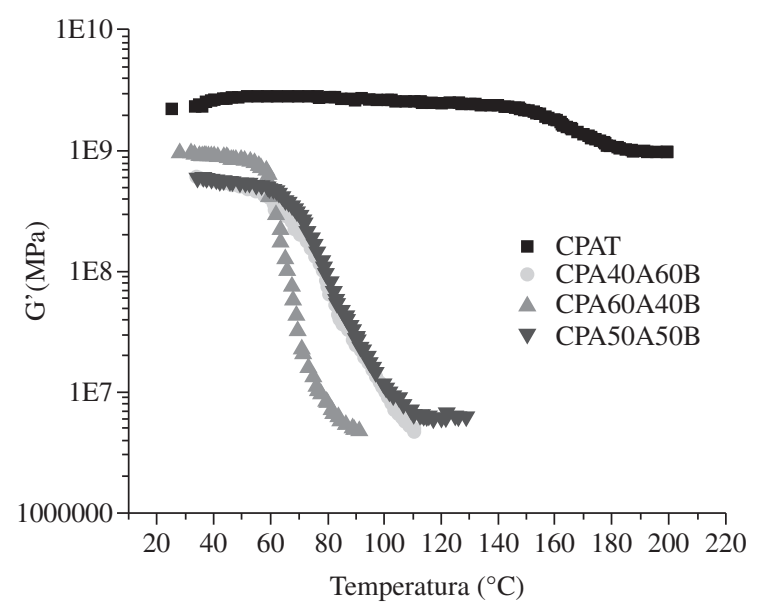

(a)

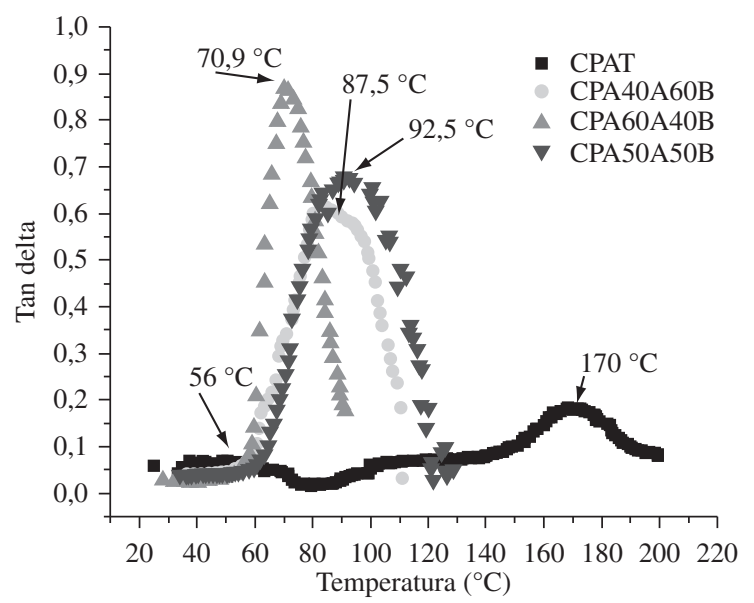

(b)

Figura 7. DMTA das amostras CPAT, CPA40A60B, CPA60A40B e CPA50A50B: a) curvas G'; e b) curvas tan delta (G”/G').

foram medidas as massas dos componentes, sendo possível verificar que a proporção da amostra CPA60A40B é a que mais se aproxima do especificado. As amostras CPA40A60B e CPA50A50B apresentaram G' iguais a 0,66 e 0,60GPa, respectivamente. No decorrer da análise, a amostra de tubo somente perde rigidez a $150{ }^{\circ} \mathrm{C}$, mantendo ainda assim $1 \mathrm{GPa}$, isto é, um valor ainda significativo, o que não ocorre com os adesivos, que perdem bruscamente a rigidez por volta de $60^{\circ} \mathrm{C}$. Da Figura 7b, é possível obter-se a temperatura de transição vítrea $\left(\mathrm{T}_{\mathrm{g}}\right)$ das amostras. Nota-se que a rigidez do tubo reduz próximo à sua temperatura de transição vítrea $\left(170{ }^{\circ} \mathrm{C}\right)$ que, por sua vez, é significativamente superior àquelas apresentadas pelos adesivos. Cabe ressaltar que as $\mathrm{T}_{\mathrm{g}}$ obtidas são similares aos resultados encontrados por Laza et al. ${ }^{[11]}$, que verificou a variabilidade deste parâmetro com a quantidade de catalisador. Dessa forma, fica evidente, a incompatibilidade termo-mecânica entre os tubos de PRFV e o adesivo.

\section{Temperatura de amolecimento de Vicat}

A Tabela 2 apresenta os resultados para o caso de carregamento de $50 \pm 1,0 \mathrm{~N}$ e taxa de aquecimento de $120 \pm 10^{\circ} \mathrm{C} / \mathrm{h}$, 
Tabela 2. Temperatura média de amolecimento de Vicat $\left(\mathrm{T}_{\mathrm{vm}}\right)$ para carregamento de $50 \pm 1,0 \mathrm{~N}$ e $120 \pm 10{ }^{\circ} \mathrm{C} / \mathrm{h}$

\begin{tabular}{lc}
\hline \multicolumn{1}{c}{ Nomenclatura } & $\mathbf{T}_{\mathbf{v m}}\left({ }^{\mathbf{}} \mathbf{C}\right)$ \\
\hline CPA40A60B & 80,1 \\
CPA50A50B & 79,3 \\
CPA60A40B & 65,3 \\
CPAT & $>250$ \\
\hline
\end{tabular}

visto que para a combinação $10 \pm 0,2 \mathrm{~N}$ e $120 \pm 10{ }^{\circ} \mathrm{C} / \mathrm{h}$ os valores de temperatura de amolecimento de Vicat foram superiores ao limite de trabalho do equipamento, ou seja, $250{ }^{\circ} \mathrm{C}$. Observa-se que a elevada rigidez do tubo de PRFV não permitiu determinar o $\mathrm{T}_{v}$ da amostra. Por outro lado, os adesivos apresentaram um $\mathrm{T}_{\mathrm{vm}}$ muito inferior ao limite do equipamento, ainda observa-se pelos resultados que com uma maior quantidade de catalisador e maior foi o $\mathrm{T}_{\mathrm{vm}}$, indicando maior rigidez a penetração com a elevação de catalisador. Estes valores indicam que nenhuma proporção de mistura de adesivo apresentou desempenho termomecânico próximo à amostra de tubo (CPAT).

\section{Conclusões}

Da avaliação preliminar por meio de espectros de FTIR, as sobreposições entre as amostras analisadas e aquelas substâncias provenientes de banco de dados estimam que o componente A do adesivo poderia ser uma resina epóxi tipo bisfenol A e o componente B um catalisador tipo 4,4' metileno bis(ciclohexilamina). As misturas de adesivo apresentaram sobreposição das bandas de absorção dos componentes individuais com diferenças apenas nas intensidades das bandas. Dos espectros de FTIR dos tubos de PRFV é possível estimar que estes podem ser compostos de uma resina epóxi curada com melamínica na relação 1:1 ou epicloridrina. Das análises termomecânicas (TGA, DMTA e Vicat) foi verificado que os adesivos iniciam degradação térmica a temperaturas inferiores àquelas apresentadas pelo tubo, sendo que na temperatura ambiente ambos apresentam desempenho satisfatório no que tange à degradação e rigidez nas aplicações em tubulações de plataformas marítimas. Contudo, foi verificada queda de desempenho a elevadas temperaturas, principalmente dos adesivos nas diferentes proporções avaliadas.

\section{Agradecimentos}

À CAPES e à PETROBRAS pelo apoio financeiro.

\section{Referências Bibliográficas}

1. Evans, K. E. \& Alderson, K. L. - Low velocity transverse impact of filament-wound pipes: Part 2. Residual pro- perties and correlations with impact damage. Composite Structures, 20, p.47-52 (1992).

2. Salibi, Z. - Performance of reinforced thermosetting resin pipe systems in desalination applications: a longterm solution to corrosion - The Arabian Gulf example. Desalination, 138, p.379-384 (2001).

3. Martens, M. \& Ellyin, F. - Biaxial monotonic behavior of a multidectional glass fiber epoxy pipe. Composites: Part A, 31, p.1001-1014 (2000).

4. Joseph, E. \& Perreux, D. - Fatigue behavior of glassfibre/epoxy-matrix filament wound pipes: tension loading tests and results. Composites Science and Technology, 52, p.469-480 (1995).

5. Gibson, A. G. - "The cost effective use of fibre reinforced composites offshore". University of Newcastle Upon Tyne, Health and Safety Executive (HSE) - Newcastle - United Kingdom (2003).

6. Brum, M. C. - "Metodologia de colagem com avaliação do comportamento de adesivos por meio de testes de tração". Dissertação de Mestrado, Universidade Federal do Rio de Janeiro, Brasil (2002).

7. ASTM D1525 - American Society for Testing and Materials. Standard Test Method for Vicat Softening Temperature of Plastics (2006).

8. Sánchez-Soto, M.; Pagés, P.; Lacorte, T.; Briceño, K. \& Carrasco, F. - Curing FTIR study and mechanical characterization of glass bead filled trifunctional epoxy composites. Composites Science and Technology, 67, p.1974-1985 (2007).

9. Delor-Jestin, F.; Drouin, D.; Cheval, P.-Y. \& Lacoste, J. - Thermal and photochemical ageing of epoxy resin Influence of curing agents. Polymer Degradation and Stability, 91, p.1247-1255 (2006).

10. Alonso, M. V.; Auad, M. L. \& Nutt, S. - Short-fiberreinforced epoxy foams. Composites: Part A, 37, p.1952-1960 (2006).

11. Laza, J. M.; Julian, C. A.; Larrauri, E.; Rodriguez, M. \& Leon, L. M. - Thermal scanning rheometer analysis of curing kinetic of an epoxy resin: 2. An amine as curing agent. Polymer, 40, p.35-45 (1998).

12. Pires, G.; Pereira, D. S.; Dias Filho, N. L. \& Vecchia, G. D. - Caracterização físico-química do sistema éster de silsexquioxano/resina epóxi DGEBA/dietilenotriamina. Revista Matéria, 20, p.317-330 (2005).

Enviado: 24/05/07

Reenviado: 20/10/07

Aceito: 29/10/07 